\title{
Mineral Candidates for Planet Interiors
}

\section{Computer simulations uncover new high-pressure minerals that may explain the origin of Earth's water and of Uranus' and Neptune's magnetic} fields.

\section{By Michael Schirber}

$$
\text { s }
$$
t takes imagination-or at least a good computer simulation-to journey to the center of a planet. Two research teams have found potential solutions to important planetary mysteries by predicting the compounds that could survive in the extreme environments of planet interiors. One

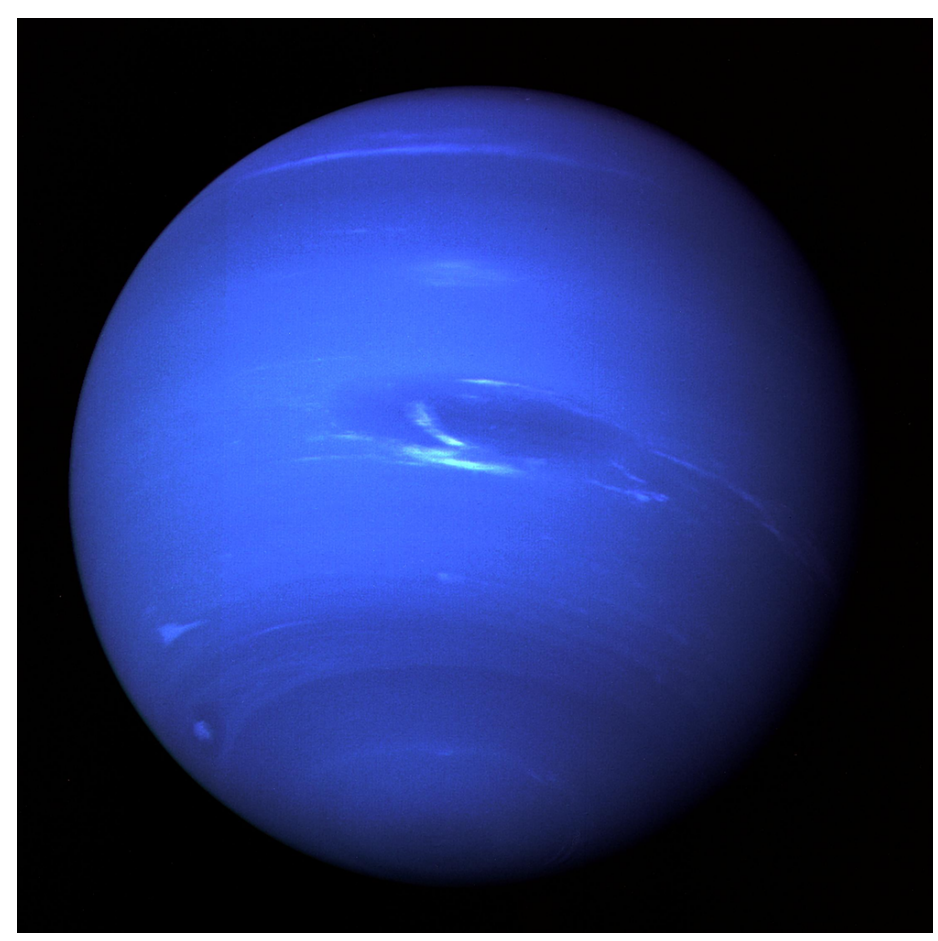

Ice planet. Simulations suggest that the magnetic fields of Neptune (shown) and Uranus could be generated by a current of protons in a silica-containing mineral in the planets' icy mantles. Credit: NASA/JPL-Caltech team has discovered a magnesium silicate that could retain water in the core of the early Earth-possibly providing the source for our planet's oceans [1]. The other team has theoretically plunged into the icy mantles of Uranus and Neptune and found new mineral candidates that could explain these planets' magnetic fields [2].

Deep inside a planet, pressures reach hundreds of gigapascals (GPa)-millions of times atmospheric pressure-and thousands of degrees kelvin. Recreating those conditions in the laboratory is very difficult, so researchers often rely on "guesses" for what planets hide in their interiors. Several groups have developed algorithms that take a set of atomic and molecular ingredients and predict stable crystal structures from these components.

Xiao Dong from Nankai University, China, and his colleagues have used one of these algorithms to explore the origin of water on Earth. Earth formed from rocky material that was too warm to hold ice, and liquid water would have evaporated away. One hypothesis is that water was delivered by ice-filled comets from the outer Solar System. However, the ratio of deuterium to hydrogen in the oceans is different from that in comets. Moreover, recent evidence suggests that the rocky material that formed Earth could have trapped water inside its crystal structure [3]. These so-called "hydrous minerals" might have temporarily stored water in Earth's interior before releasing it to the surface. One challenge for this hypothesis is identifying hydrous minerals that would have been abundant inside Earth.

Dong and his colleagues explored whether silicates-a common type of mineral in Earth's interior-could hold water. Unlike 


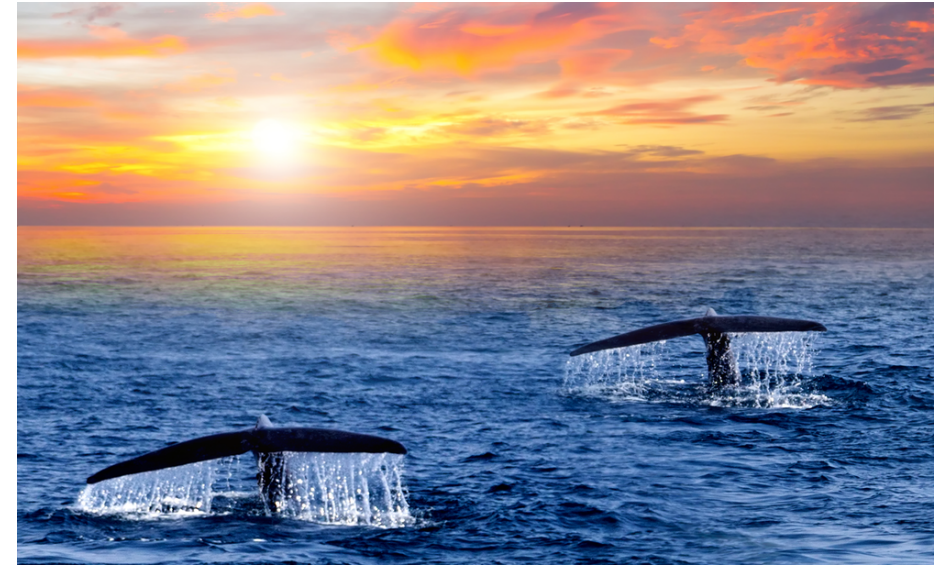

Water, water, everywhere. Oceans cover a majority of Earth's surface, but the source of our $\mathrm{H}_{2} \mathrm{O}$ is not known. New simulations suggest that large volumes of rock made of a silicate mineral from Earth's core could have gradually risen toward the surface and released enough water to fill the oceans.

Credit: stock.adobe.com/Emoji Smileys People

previous researchers, they considered pressures that would be relevant in Earth's core: 136 to $364 \mathrm{GPa}$. The core mostly consists of iron alloys now, but silicates could have been present long ago. "We realized that in the early Earth, things were quite different, and silicates would have existed all the way down to the planet's center," Dong says. To investigate these core silicates, the researchers plugged four elements-magnesium (Mg), silicon (Si), oxygen (O), and hydrogen $(\mathrm{H})-$ into their crystal structure algorithm. They discovered a new crystalline structure, $\mathrm{Mg}_{2} \mathrm{SiO}_{5} \mathrm{H}_{2}$, which only exists at pressures greater than $260 \mathrm{Gpa}$. At lower pressures, it decomposes into $\mathrm{MgO}, \mathrm{MgSiO}_{3}$, and $\mathrm{H}_{2} \mathrm{O}$.

This high-pressure silicate is $11 \%$ water by weight, which is comparable to other hydrous minerals. Given the abundance of magnesium and silicon in Earth's interior, the team estimates that $\mathrm{Mg}_{2} \mathrm{SiO}_{5} \mathrm{H}_{2}$ could have stored as much as twice Earth's current water supply in the young planet's core. The researchers postulate that-as the planet aged-dense iron alloys settled into the core, pushing the silicates upward into lower pressure regions where they released their water. This freed water eventually trickled up to the surface and filled the oceans.

Jian Sun from Nanjing University, China, and his colleagues took on another planetary mystery: the interior composition of Uranus and Neptune. The predominant theory is that these planets have thick, icy mantles made up of frozen forms of water, ammonia, and methane. But atmospheric observations of these planets and models of planetary formation suggest that the interiors may be dominated by silicate rocks [4]. The main sticking point for the rocky interior hypothesis is how to explain the magnetic fields of Uranus and Neptune-to generate the fields, electric currents must flow inside the planet. Sun and colleagues have a possible solution involving a mixture of rock and ice that is an electric conductor.

"We have used our newly developed crystal structure search techniques and $a b$ initio molecular dynamics simulations to study the phase diagrams of the Si-O-H system," Sun says. He and his colleagues found that-at pressures above 450 $\mathrm{GPa}$-silica can react with water and hydrogen to form three compounds: $\mathrm{SiOH}_{2}, \mathrm{Si}_{2} \mathrm{O}_{5} \mathrm{H}_{2}$, and $\mathrm{SiO}_{2} \mathrm{H}_{2}$. The last two compounds exhibit a so-called "superionic" phase in which protons (hydrogen nuclei) are able to diffuse within the mineral's crystal framework. The freely moving protons would carry current and thus could generate Uranus' and Neptune's magnetic fields.

In addition, the proposed mixing of silica rock with ice and hydrogen could make the core-mantle boundary in these ice giants less sharp, as expected in "fuzzy" core models [5]. To confirm these predictions, Sun says future experiments could try to produce these compounds by using high-powered lasers or electric field pulses to create the high-pressure conditions.

"The results [from both teams] are new, with potentially very important implications," says astronomer Tristan Guillot from the Côte d'Azur Observatory in France. He says the researchers address a long-standing problem over how elements mix within planetary interiors. In particular, the proposed mixing of silica and water in Uranus and Neptune "has strong consequences for heat transfer in these planets and therefore on their structure, evolution, and magnetic-field generation," Guillot says.

The origin of Earth's water is one of many open questions about our planet's formation, says planetary scientist Ravit Helled from the University of Zurich. "We still don't know exactly how much water Earth has in its deep interior today," she says. If the core served as an important water reservoir-as suggested by 
Dong and colleagues-then similar water storage may have occurred in other rocky planets, affecting how they evolved, Helled says.

Michael Schirber is a Corresponding Editor for Physics based in Lyon, France.

\section{REFERENCES}

1. H. Li et al., "Ultrahigh-pressure magnesium hydrosilicates as reservoirs of water in early Earth," Phys. Rev. Lett. 128, 035703 (2022).
2. H. Gao et al., "Superionic silica-water and silica-hydrogen compounds in the deep interiors of Uranus and Neptune," Phys. Rev. Lett. 128, 035702 (2022).

3. L. Piani et al., "Earth's water may have been inherited from material similar to enstatite chondrite meteorites," Science 369, 1110 (2020).

4. N. A. Teanby et al., "Neptune and Uranus: ice or rock giants?" Philos. Trans. R. Soc. A 378, 20190489 (2020).

5. R. Helled et al., "Uranus and Neptune: Origin, Evolution and Internal Structure," Space Sci. Rev. 216, 38 (2020). 